\title{
Impact of Yeast Glycosylation Pathway on Cell Integrity and Morphology
}

\author{
Anna Janik, Mateusz Juchimiuk, Joanna Kruszewska, Jacek Orłowski, \\ Monika Pasikowska and Grażyna Palamarczyk
}

Additional information is available at the end of the chapter

http://dx.doi.org/10.5772/48102

\section{Introduction}

The subject of protein $\mathrm{N}$ - and O-glycosylation in the yeast S.cerevisiae has already been well described in many excellent reviews [1-3]. However, less is known about the occurrence of these processes in the yeast Candida albicans, an opportunistic human pathogen.

In the $\mathrm{N}$-glycosylation reactions catalysed by the Alg enzymes, dolichyl phosphate (DolP) serves as a lipid acceptor of sugar residues. The Alg enzymes are present both on the cytosolic side of the ER: Alg7, Alg1, Alg2 and Alg11) and on its lumenal face(Alg3, Alg5, Alg6, Alg8-10 and Alg12 [3-6]. Cytosolic Alg enzymes, necessary for viability of yeast and mammalian cells in culture, produce DolPP-GlcNAc2Man5 from DolP, UDPGlcNAc and GDPMan. There is also circumstantial evidence that DolPP-GlcNAc2Man5 is delivered into the lumen of the ER by a putative flippase (Rft1p) [7]. Within the ER the DolPPGlcNAc2Man 5 product is converted to DolPP-GlcNAc2Man9Glc3 using DolPMan and DolPGlc as sugar donors [3]. As it was mentioned already DolPMan is also a substrate for protein $O$-glycosylation, where it serves as a donor of the first mannose to be attached to hydroxyl groups of serine and threonine. The second and subsequent mannose residues are transferred directly from GDPMan [1,2]. DolPMan is also involved in the synthesis of the sugar part of glycosylphosphatidyl inositol anchor in yeast and other eukaryotes [2]. Moreover, a large group of cell wall glycoproteins is attached to the glucan polymers via a GPI-remnant structure.

On the other hand, there is a growing literature describing the involvement of cell wall carbohydrates in fungus-host interactions (for review see [8]), as well as in the maintenance of cell wall integrity [9]. Thus, one can predict a functional link between N-glycosylation and O-mannosylation of cell wall proteins, cell wall integrity and/or fungus-host interactions. Nonetheless, open questions remain concerning the regulatory mechanisms of early events of 
protein glycosylation and their impact on the synthesis of outer glycan chain in cell surface glycoproteins of S. cerevisiae and C. albicans.

A model of the $S$. cerevisiae cell wall has been described. It contains four classes of interacting components: chitin, $\beta 1,3$ and $\beta 1,6$ glucan and mannoproteins $[10,11]$. This structure is similar to that of the C. albicans cell wall [11-13].

\section{Contribution of the mevalonate pathway to protein glycosylation and cell wall integrity}

Mevalonate pathway in yeasts is important not only for ergosterol biosynthesis but also for the production of nonsterol molecules, deriving from farnesyl diphosphate. Formation of cell wall proteins, i.e. the glycosyl- phosphatidylinositol (GPI) anchored (GPI-CWP) and proteins with internal repeats (pir-CWP,) requires, as an initial intermediate, DolP synthesized together with the other isoprenoid lipids in the mevalonate pathway.

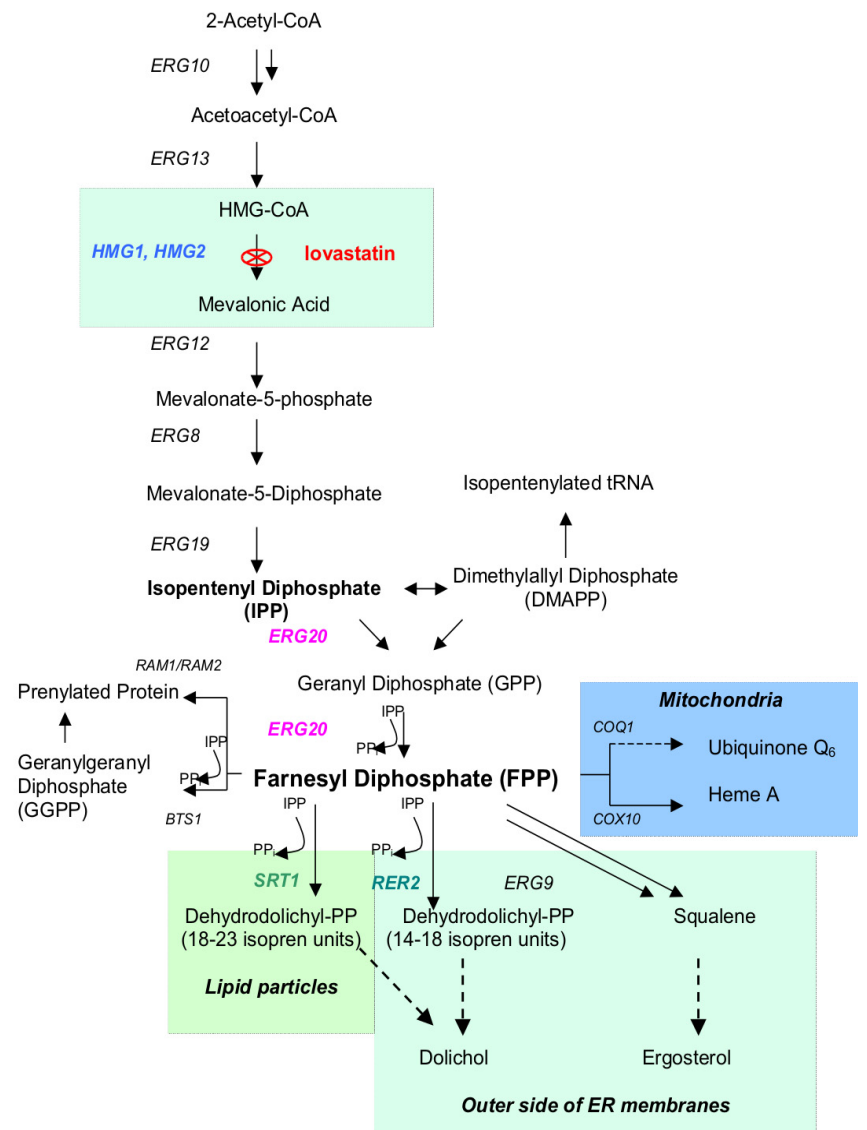

Figure 1. Isoprenoid (mevalonate) pathway in the yeast Saccharomyces cerevisiae. 
Dolichol biosynthetic pathway has multiple levels of regulation. Thus, its cellular level is amenable to alterations affecting protein glycosylation and, in consequence, the cell wall structure. The pathway is shared with other isoprenoid lipids (Fig.1). The most abundant branch of the pathway, leading to sterol biosynthesis, is one of the main targets for antifungal drugs, which exploit the differences in the pathways and the end product - ergosterol in fungal cells and cholesterol in animals. The mevalonate pathway diverges after the synthesis of farnesyl diphosphate by farnesyl diphosphate synthase (FPPS), encoded by the ERG20 gene.

Products of the subsequent reactions are shown together with the names of the genes, in capital letters, encoding the enzymes catalyzing them. Enzyme affected by lovostatin, an inhibitor of isoprenoid pathway, is indicated (K. Grabinska PhD thesis, IBB 2002)

Using a yeast based two hybrid system, we have identified the Yta7 protein interacting with FPPS, and showed that it was membrane-associated and localised both to the nucleus and the endoplasmic reticulum (ER). In order to assess the importance of the mevalonate pathway for cell wall synthesis and its role in cell-wall integrity (Fig 2), we investigated the effects of YTA7 deletion at the transcriptional level. Our data [14] show that loss of YTA7 function leads to activation of the genes implicated in cell wall integrity pathway (CRZ1, FKS2, and KNR4) and highlight a possible link between dolichol metabolism and cell wall synthesis.

Moreover, farnesol, which is likely to be derived from dephosphorylation of FPP, inhibits growth of $S$. cerevisiae and C. albicans. This inhibition is concomitant with a significant loss of intracellular diacylglycerol (DAG) [15], which is an activator of the PKC1-signaling pathway involved in the maintenance of cell wall integrity (described later, compare Fig.3)

De novo synthesis of dolichol starts with the 1'-4 condensation of farnesyl diphosphate (FPP) with 11-15 isopentenyl pyrophosphate units to form polyprenyl diphosphate (dehydrodolichol diphosphate) (Fig. 2). This reaction is catalysed by cis-prenyltransferase (cis-Ptase) encoded in S. cerevisiae by the RER2 and SRT1 genes [16].

Our results indicate that FPP or its derivatives regulate the transcription of RER2 and SRT1 genes as well as of DPM1, which encodes Dpm1p [17]. To enter the glycosylation pathways the RER2 and SRT1 gene products (dehydrodolichol diphosphate) need to be dephosphorylated, reduced and phosphorylated again by CTP-dependent dolichyl kinase, Sec59p [18-20].

Biosynthesis of isopentenyldiphosphate (IPP) from mevalonate diphosphate is catalysed by mevalonate pyrophosphate decarboxylase (Fig.1). Subsequently IPP is converted to dimethylallyl diphosphate (DMAPP) and DMAPP ( $\left.\mathrm{C}_{5}\right)$ is condensed with farnesyl diphosphate, composed of 3 isoprene units $\left(\mathrm{C}_{15}\right)$. This reaction is catalysed by Rer 2 and Srt1p. Further elongation of the isoprenol chain occurs, by step wise addition of the 5-carbon isoprene units to reach species-specific chain length. Poliprenol diphosphate is dephosphorylated and reduced by alfa saturase (Dfg10p) to form dolichol. To enter glycosylation pathway dolichol is phosphorylated by CTP-dependent dolichol kinase. 


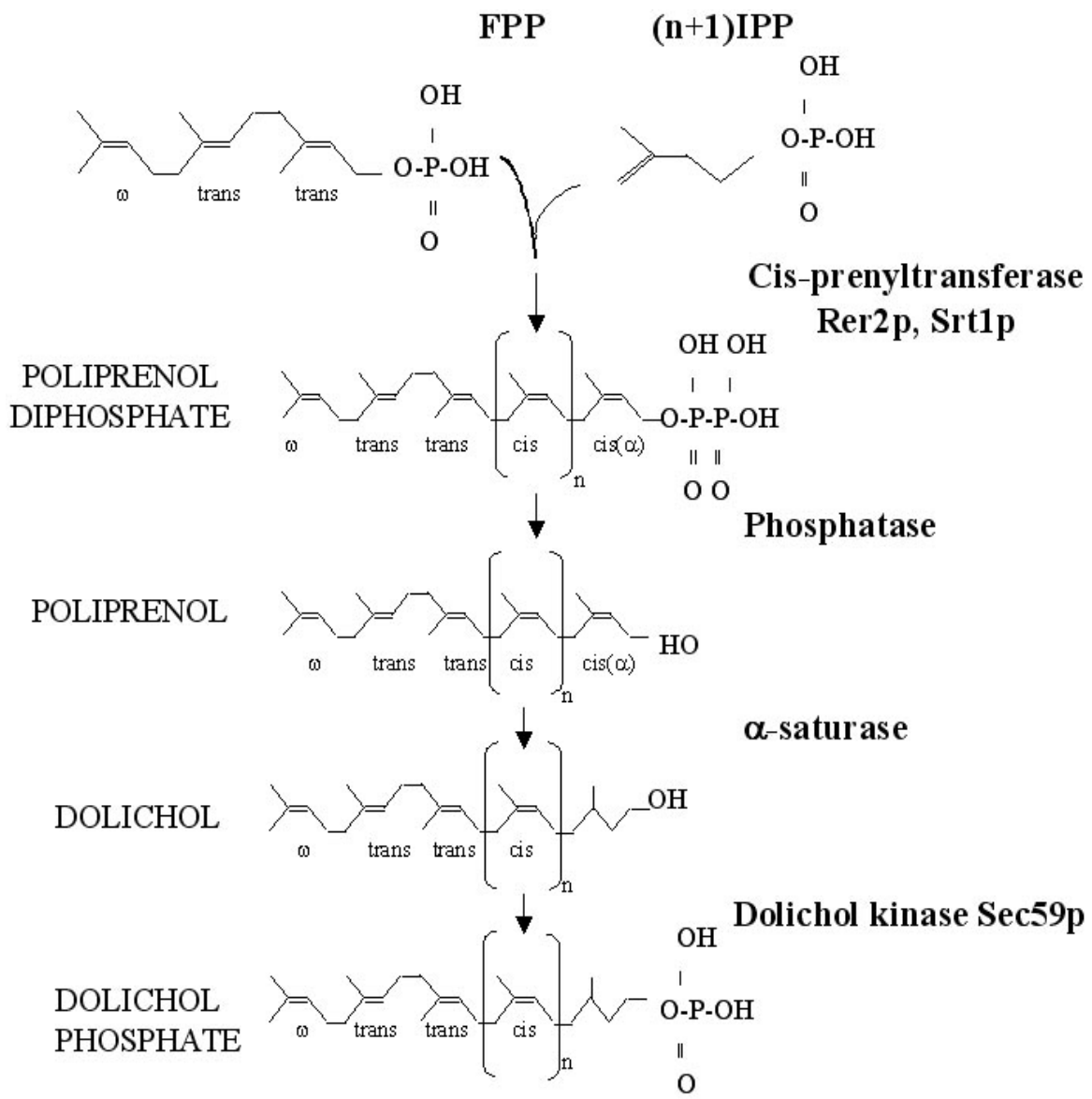

$\mathrm{n}=10-14$ isoprene units for Rer2p and 14-23 for Srt1p products. (Adopted from [21])

Figure 2. Dolichyl phosphate biosynthesis de novo in yeast.

Our data indicate that in the rapidly dividing yeast cells "de novo" biosynthesis of dolichyl phosphate described above is a main source of its supply.

However, it is thought that in non dividing cells dolichyl phosphate might be derived mainly from recycling after each cycle of protein glycosylation, either by transfer of the oligosaccharide from DolPPGlcNAc2Man9Glc3 onto acceptor protein or from the transfer across the ER membranes of single glucose (Glc) and mannose (Man) residues from DolPMan or DolPGlc [22].

The length of dolichol molecules is species-specific and in yeast contains 14-18 isoprene units [23]. Although a great deal of progress has been made in the understanding of the enzymatic steps responsible for polyprenyl chain length termination and conversion of dehydrodolichol to dolichol, some open questions still remain. In vitro, cell extracts and 
membrane fractions from $S$. cerevisiae catalyse the biosynthesis of dolichol backbone i.e. dehydrodolichol [24] whereas in vivo yeast synthesise dolichols. On the base of the results obtained so far it was assumed that in S. cerevisiae polyprenyl diphosphates synthesised in vitro undergo immediate dephosphorylation and reduction of its alpha residue [24, 25]. A similar conclusion was reached for the rat liver system [26] and in general for metazoan eukaryotic cells [22].

\section{Cell wall alteration resulting from the defect in dolichol and dolichyl phosphate formation}

It has been shown that depletion of GDPMan pyrophosphorylase activity in C.albicans [27] leads to cell lysis, inefficient cell separation, impaired bud growth, clumping and flocculation, as well as increased sensitivity to a wide range of antifungal drugs. GDPMan pyrophosphorylase ( $C a$ SRB1 encoded) catalyses the transfer of Man-1P to GDP and is responsible for the biosynthesis of GDPMan, the major sugar donor in yeast and filamentous fungi. On the other hand, overexpression of GDPMan pyrophosphorylase encoding gene (mpg1) in the filamentous fungus Trichoderma reesei resulted in a two-fold increase in GDPMan level, overglycosylation of secreted proteins, increased transcription of dpm1, DolPMan-synthase encoding gene, and increased enzyme activity [28]. Thus, it was assumed that the cellular level of GDPMan is one of the factors playing a regulatory role in protein glycosylation.

In this work we have concentrated on the assembly of mono- and oligo-saccharide lipid carrier (DolP), which is another substrate in protein glycosylation, and on it's effect on cell wall integrity and cell morphology.

In C.albicans, which unlike S.cerevisiae is an obligatory diploid, we initially cloned the genes involved in dolichol biosynthesis (CARER2 CaSRT1) and phosphorylation (CASEC59). To construct mutants defective in Rer2p or Sec59p activities, we used "URA-blaster" cassette [29] to delete one chromosomal copy of the gene. Since the genes under investigation were either essential or generated mutants with strong phenotypes, we adopted a conditional mutant approach and the second copy of the gene was placed under the control of a regulatable promoter.

By growing the strains in repressive conditions we were able to demonstrate that the defect in dolichol backbone synthesis or its phosphorylation, resulted in the aberrant cell wall structure and increased sensitivity to some antifungal drugs. Moreover, the normal morphogenesis of the fungus, e.g. hyphae formation, was prevented (Juchimiuk et al. 2011, in preparation).

Recently, we have cloned an ortholog of S.cerevisiae DFG10 from C.albicans (orf 19.7841). S.cerevisiae DFG10 encodes a protein with 3-oxo-5-alpha-steroid 4-dehydrogenase activity, lately postulated to be involved in dehydrodolichol reduction [30]. The latter was based on the finding that the mutant, named $d f g 10-100$, isolated in a genetic screen for the strains defective in filamentous growth [31], was shown to be defective in N-linked glycosylation. 
This defect was conferred by orf encoding the human SRD5A3 protein, involved in the reduction of dehydrodolichol diphosphate into dolicholdiphosphate. Thus, it was assumed that SRD5A3 is the human ortholog of the yeast DFG10 gene encoding dehydrodolichyl diphosphate reductase. The product of DFG10 shows $25 \%$ amino acid identity and $43 \%$ similarity with the human SRD5A3 protein, forming with others SRD proteins the steroid 5a-reductase family [30].

Double deletion of the C.albicans orf19.7841 rendered viable Cadfg10 mutant strain. Its biochemical analysis revealed that the strain produces $70 \%$ of dehydro- and $30 \%$ of dolichols, whereas in the wild type, parental strain only dolichols are synthesized. The mutant strain was also oversensitive to tunicamycin, an inhibitor of N-glycosylation. Indeed, a defect in protein glycosylation was confirmed by assessing the degree of glycosylation of the marker glycoprotein hexosaminidase. Moreover, the Cadfg10 mutant exhibits abnormal hyphal growth and increased resistance to some antifungal agents, thus indicating alterations of the C.albicans cell wall (Janik et al., manuscript in preparation).

However, based on our results, only $30 \%$ of dehydrodolihol is reduced to dolichol in Cadfg10 null mutant. Thus, it is possible that CaDfg10p is not the only protein involved in dehydrodolichol reduction. Another likely candidate is an ortholog of S.cerevisiae TSC 13, encoding steroid reductase, i.e. putative enoyl reductase, encoded in C.albicans by orf 19.3293. However, the possible role of orf 19.3293 in dolichol synthesis is now under investigation.

We have also studied alterations in cell wall composition and integrity in S. cerevisiae dpm1-1 mutant impaired in DolPMan formation and in the sec59 mutant impaired in dolichol kinase activity. In our earlier work we were able to demonstrate that overexpression of the S.cerevisiae DPM1 gene, encoding DolPMan synthase in T.reesei and Aspergillus nidulans led to increased secretion of fully glycosylated proteins, concomitant with alteration of the cell wall ultrastructure (T.reesei) or with accumulation of overproduced glycoproteins in the periplasmic space (A.nidulans) [32,33].

For the S. cerevisiae dpm1-1 as well as for the sec59-1 mutants we observed an increased sensitivity to Calcofluor White (CFW) and an upregulated chitin level. Both mutated strains were also oversensitive to a variety of the external agents including antifungal drugs [34].

We have shown that the sec59 and dpm1 mutants are affected in cell wall composition [35]. A search for multicopy suppressors of the mutant phenotype, resulted in the isolation of the RER2 gene, which as described above, is involved in the synthesis of the dolichol backbone and enhances protein glycosylation. In addition, the sec 59-1 phenotype could be rescued by overexpression of the ROT1 gene, encoding the endoplasmic reticulum Rot1 protein. The latter is implicated in cell wall biogenesis and acts as a chaperone for misfolded proteins [36, 37]. Recently, we have shown also that Rot1 interacts with Ost3, one of the nine subunits of the oligosaccharyltransferase complex, the key enzyme of $N$-glycosylation. Deletion of OST3 in the rot1-1 mutant causes a temperature sensitive phenotype as well as sensitivity towards compounds interfering with cell wall biogenesis, such as Calcofluor White, caffeine, Congo Red and hygromycin B. Oligosaccharyltransferase activity determined in vitro in membranes 
from rot1-1ost3 $\Delta$ cells was found to be decreased to $45 \%$ compared to wild-type membranes, and model glycoproteins of $N$-glycosylation, like carboxypeptidase CPY, Gas1 or DPAP B, displayed an underglycosylation pattern. A physical interaction between Rot1 and Ost3 was demonstrated by affinity chromatography. Moreover, Rot1 was also found to be involved in the $O$-mannosylation process, as glycosylation of distinct glycoproteins of this type was affected as well. Altogether, it can be assumed that Rot1 acts also as a chaperone required to ensure proper glycosylation [38].

As already mentioned, a defect in protein O-mannosylation in fungi results in impaired cell wall integrity [9]. This process is initiated at the luminal side of the ER. The key enzyme of O-mannosylation is protein -O-mannosyltransferase (Pmtp) catalysing direct transfer of Man from DolPMan into the serine/threonine $\mathrm{OH}$ group in acceptor protein. This is followed by the addition of a short linear glycan, composed of mannosyl residues, directly from GDPMan. Whereas O-mannosylation is initiated in the ER, further modifications of the glycan chain occur in the Golgi apparatus. In S. cerevisiae the Pmt proteins are encoded by seven PMT genes [39]. A family of PMT genes was also identified in C. albicans [40] and in another fungal pathogen Cryptococcus neoformans [41]. Phylogenetic analysis of Pmt proteins revealed that they can be grouped in three subfamilies, Pmt1p, Pmt2p and Pmt4p [42]. The functions of Pmt proteins were studied, and it was demonstrated that in S.cerevisiae Omannosylation affects protein stability, localisation and transport from the ER [43]. In C.albicans O-mannosylation is important for morphogenesis, adherence to host cells and virulence [44]. Characterization of the PMT gene family in C. neoformans revealed that Pmt proteins play a crucial role in maintaining cell morphology and cell wall integrity [41]. Similarly, in the filamentous fungus T.reesei diminished overall activity of Pmt proteins resulted in decreased $\mathrm{O}$ - as well as $\mathrm{N}$-glycosylation and aberrant cell wall composition [45].

Studies of the effect of tunicamycin revealed the effect of the dolichol dependent protein Nglycosylation on C. albicans biofilm development. In its normal niche C.albicans forms biofilms that are attached to cell surfaces. In addition to the mucosal surfaces, such biofilms are often formed on implanted medical devices [46, 47]. Fully mature C.albicans biofilms consist of a complex of yeast, hyphae and pseudohyphae and exhibit increased resistance to antifungal drugs $[48,49]$. Tunicamycin is a nucleoside antibiotic, produced by Streptomyces lysosuperficus that blocks the transfer of GlcNAc-1-P from UDPGlcNAc to DolP. This reaction, catalysed by the Alg7 protein, initiates the formation of DolPP-oligosaccharide and hence the whole $\mathrm{N}$-glycosylation process. It has been demonstrated that physiological concentrations of tunicamycin display a significant inhibitory effect on biofilm development and maintenance, without affecting overall cell growth or morphology. Based on the above, conclusion was reached underlying the role of $\mathrm{N}$-glycosylation in the developmental stages of biofilm formation [50]

\section{Cell morphology in Golgi glycosylation mutants}

A number of data indicates that a defect in glycosylation process occurring in the Golgi stack might affect cell morphology and virulence. 
Initial steps of protein $\mathrm{N}$ - and O-glycosylation described so far occur in the ER. "Dolicholdependent" glycosylation ends with the formation of DolPP-oligosaccharide (DolPPGlcNAc2Man9Glc3) and subsequent transfer of the oligosaccharide to the beta amido group of asparagine within the $\mathrm{N}$-glycosylation site (Asn/X/Ser). Such a glycosylated peptide undergoes partial trimming, which is species-specific and in yeast involves removal of the three Glc and one Man residues. Partially processed glycopeptide is transported to the Golgi stack where the saccharide part (GlcNAc2Man8) undergoes further processing and maturation. Whereas glycosylation reactions occurring in the ER are well conserved in the eukaryotic cells, N-glycan processing in the Golgi is greatly diverse. In yeast, the core structure of glycoproteins is hypermannosylated (up to 200 Man residues), forming a backbone made up of alpha 1,6 linked residues, branched by alpha 1,2- and alpha 1,6 mannosyl residues. In addition, another mature core structure occurs in the Golgi, i.e. GlcNAc2Man8-13 [51]. A number of Golgi mannosyltransferases is involved in the synthesis of the sugar backbone and branching. It offers a vast majority of modifications of the sugar structure. The OCH1 gene encodes an alpha 1,6 mannosyltransferase initiating addition of the outer sugar chain (www.yeastgenome.org). Deletion or depletion of OCH1 causes lethality or slow-growth. The och1 mutants cannot form high mannose oligosaccharides ( 50+ Man residues) so they have decreased levels of cell wall mannoproteins, causing weakness and defects in bud formation and hypersensitivity to agents that attack the cell wall (calcofluor white, hygromycin B and SDS). Weakening of cell walls in hypotonic solution can be partially suppressed by the addition of osmotic stabilizers such as salt or sorbitol.

Mannan branching involves Mnn1p, one of five S. cerevisiae proteins of the MNN1 family. It is alpha-1,3-mannosyltransferase, an integral membrane glycoprotein of the Golgi complex, required for addition of alpha1,3-mannose linkages to $\mathrm{N}$ - and O-linked oligosaccharides,. Mnn1 mutant exhibits decreased sensitivity to hygromycin B [52]. It has been demonstrated that a double deletion of the OCH1 and MNN1 genes renders a strain which is unable to grow at nonpermissive temperature and is defective in cell cytokinesis. On the other hand, no clear effect on the cell integrity was observed [53]. However, the role of Mnn5 protein (an iron regulated alpyha-1,2 mannosyltransferase) from C.albicans in cell wall integrity maintenance was demonstrated [54]. A mnn5 delta mutant contains a reduced amount of cell wall mannan and hypersensitivity to the cell wall damaging agents. Disruption of the $\mathrm{C}$. albicans OCH1 homologue resulted in a temperature-sensitive growth defect and cellular aggregation. Outer chain elongation of $\mathrm{N}$-glycans was absent in the null mutant, demonstrated by the lack of the alpha 1,6linked polymannose backbone and the underglycosylation of $N$-acetylglucosaminidase. Moreover, the null mutant was hypersensitive to a range of cell wall perturbing agents. These mutants had near normal growth rates in vitro but exhibited decreased virulence in a murine model of systemic infection. Based on this the importance of $\mathrm{N}$-glycan outer chain epitopes to the host-fungal interaction and virulence was assumed $[55,56]$.

\section{Evidence for the role of glycan in cell defence mechanisms}

The fungal cell wall is a highly dynamic structure, essential for the shape and stability of the cell. Thus in yeast and fungi cell wall integrity is tightly controlled by the activation of the protein kinase $\mathrm{C}$-dependent MAP-kinase pathway [57] (Fig. 3). 


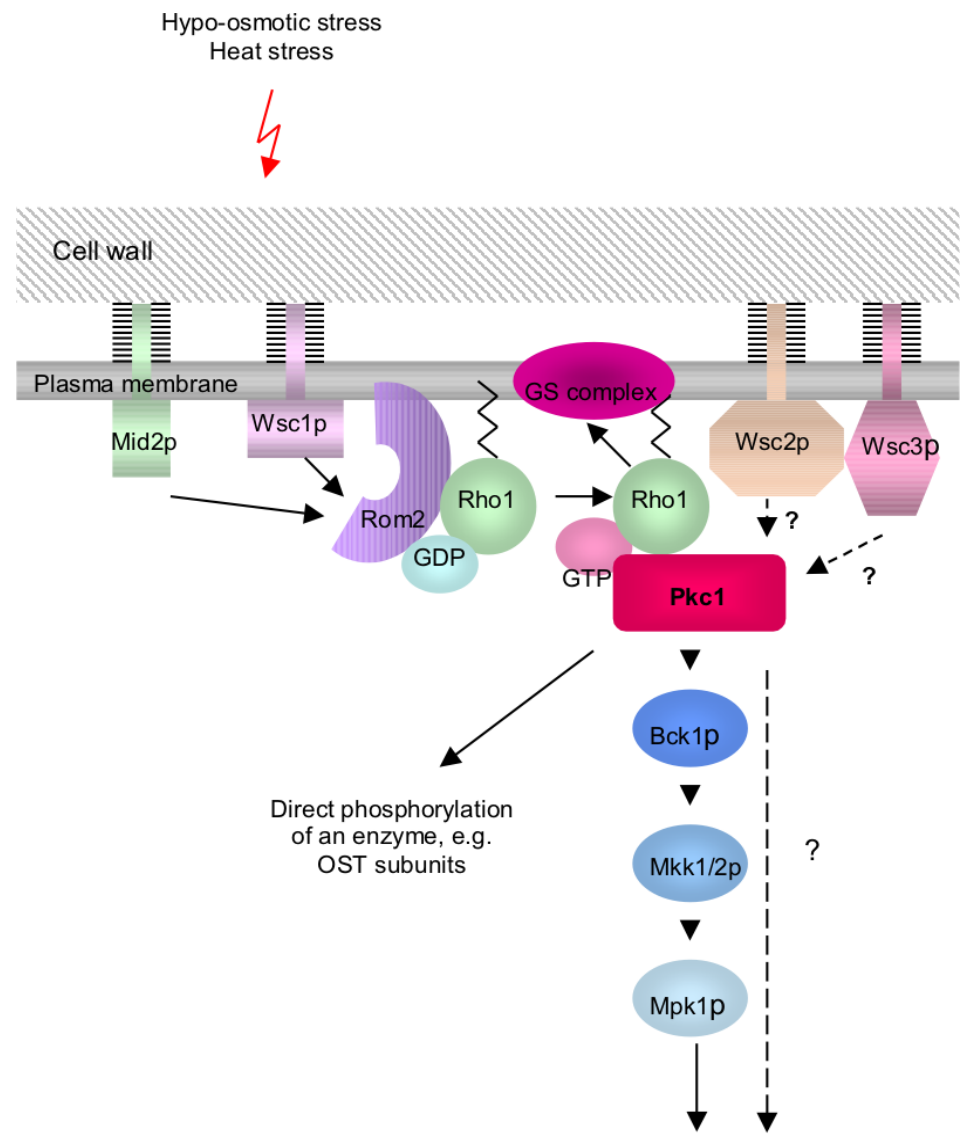

Transcription activation

Figure 3. Cell wall integrity pathway (CWIP) in Saccharomyces cerevisiae

The sensors of the cell wall damage located in the plasma membrane (Mid2p, Wsc1-3p) pass the signal thorough the Rom1p to Rho1p, which in turn activates the Pkc1p- dependent cascade of MAP kinases (Bck1p, Mkk1/2p) Mpk1 kinase activates its main targets Swi4/6p and Rlm1p, transcription factors that activate expression of the cell wall genes (adopted from [57]).

Efficiency of this process depends, among others, on the glycosylation status of the receptor proteins located in the plasma membrane [58]. It has been demonstrated that the plasma membrane protein Mid2, a putative mechanosensor, responds to cell wall stresses and changes in the cell morphology induced by pheromone treatment. The response is related to the glycosylation status of the Mid2 protein, which is highly O-glycosylated and contains two potential glycosylation sites, one of them at Asn-35, carrying N-linked sugars. It was demonstrated that O-glycosylation is responsible for the stability of the protein, whereas the 
presence of the $\mathrm{N}$-linked sugar chain is a prerequisite for its function as a sensor of external stimuli.

Our data [35] indicate that impairement of dolichol kinase (Sec59p) activity is concomitant with a defect in plasma membrane/cell wall Gas1p glycosylation and the activation of the CWIP. These results were further confirmed by the analysis of the cell wall composition of the sec59-1 mutant as compared to the parental wild type. The cell wall of the mutant strain contains a significantly increased amount of chitin and beta-1,6 glucan (2.7 and 1.7 fold, respectively). Simultaneously we observed an activation of the CWIP in sec59-1, as compared to the wild type cells. All these differences were abolished by overexpression of the suppressor RER2 gene, leading to increased synthesis of the oligosaccharide carrier (DolP) and protein glycosylation.

An activation of the cell defence mechanisms was also observed in C.albicans OCH1 mutants, affected in outer sugar chain elongation (compare above).

Together, based on the knowledge acquired so far, it can be assumed that the glycosylation pathway in yeast and fungi offers many levels of regulation which might influence the final quality and quantity of cell wall glycoproteins and consequently cell surface immunogeneity and the fungal-host interaction. This includes also the processes occurring in the ER i.e. dolichol biosynthesis and glycosylation steps involving dolichol.

\section{Author details}

Anna Janik, Mateusz Juchimiuk, Joanna Kruszewska, Jacek Orłowski, Monika Pasikowska and Grażyna Palamarczyk* Institute of Biochemistry and Biophysics, Polish Academy of Sciences, Laboratory of Fungal Glycobiology, Warsaw, Poland

\section{Acknowledgement}

The experimental work was supported by the grant N N303 577238 from Ministry of Science and Higher Education, Poland, to G.P.

\section{References}

[1] Tanner W, Lehle L. Protein glycosylation in yeast. . Biochim Biophys Acta 1987; 906, :8199.

[2] Herscovics A., Orlean P Glycoprotein biosynthesis in yeast. FASEB J. 71993; 540-550

[3] Burda P., Aebi, M. The dolichol pathway of N-linked glycosylation, Biochim. Biophys. Acta 1999;1426, 239-257

\footnotetext{
${ }^{*}$ Corresponding Author
} 
[4] Cippolo J.F., Trimble R.B., Chi J.H., Yan Q., Dean N. The yeast ALG11 gene specifies addition of the terminal alpha 1,2 Man to Man5GlcNAc2PPDolichol intermediate formed on the cytosolic side of the endoplasmic reticulum J. Biol. Chem 2001; 276, 4267-4277

[5] Huffaker T., Robbins PW. Yeast mutants deficient in protein glycosylation, Proc. Natl. Acad Sci USA 1983; 80, 7466-7470

[6] Reiss G., te Heesen S., Zimmerman J., Robbins P.W., Aebi M. Isolation of the ALG6 locus of Saccharomyces cerevisiae required for glucosylation of the N-linked glucosylation pathway. Glycobiology.1996;6:493-498

[7] Hellenius A., Aebi M. Intracellular functions of N-linked glycans 2001; Science 291, 2364-2369

[8] Masuoka J. Surface glycans of Candida albicans and other pathogenic fungi: physiological roles, clinical uses and experimental challenges. Clin. Microbiol Rev 2004; $17,281-310$

[9] Strahl-Bolsinger, S., Gentzsch, M., Tanner W. Protein O-mannosylation. Biochim. Biophys. Acta 1999; 1426, 297-307.

[10] De Nobel H., Sietsma J.H., Van Den Ende, H., Klis, F. Model organisation and construction of the fungal cell wall in The Mycota VIII. 2001; Biology of the Fungal Cell, Howard/Gow Eds. Springer-Verlag, Berlin Heidelberg pp181-200.

[11] Klis, F. M., P. De Groot, and K. Hellingwerf Molecular organisation of the cell wall of Candida albicans. Med. Mycol 2001; 39,S1,1-8.

[12] Mormeneo S., Marcilla A., Iranzo M., Sentandreu R. Structural mannoproteins released by ßelimination from Candida albicans cell wall. FEMS Microbiol. Lett 1994; 123, 131-136.

[13] Kapteyn, J. C., L. L. Hoyer, J. E. Hecht, W. H. Müller, A. Andel, A. J. Verkleij, M. Makarow, H. Van Den Ende, and F. M. Klis The cell wall architecture of Candida albicans wild-type cells and cell wall-defective mutants. Mol. Microbiol 2000; 35:601-611.

[14] Kuranda K, Grabinska K, Berges T, Karst F, Leberre V, Sokol S, François J, Palamarczyk $\mathrm{G}$ The YTA7 gene is involved in the regulation of the isoprenoid pathway in the yeast Saccharomyces cerevisiae. FEMS Yeast Res 2009; 9:381-390.

[15] Machida K, Tanaka T Farnesol-induced generation of reactive oxygen species dependent on mitochondrial transmembrane potential hyperpolarization mediated by $\mathrm{F}(0) \mathrm{F}(1)-\mathrm{ATPase}$ in yeast. FEBS Lett.1999; 462(1-2):108-12

[16] Sato, M., Sato, K., Nishikawa, Si., Hirata, A., Kato, Ji., Nakano, A The yeast RER2 gene, identified by endoplasmic reticulum protein localization mutations encodes cisprenyltransferase, a key enzyme in dolichol biosynthesis. Mol Cell Biol 1999; 19: 471483

[17] Grabińska K, Sosińska G, Orłowski J, Swiezewska E, Berges T, Karst F, Palamarczyk G Functional relationships between the Saccharomyces cerevisiae cis-prenyltransferases required for dolichol biosynthesis. Acta Biochim Pol 2005; 52: 221-32.

[18] Adair W.L., Cafmeyer N Cell cycle dependence of dolichol phosphate biosynthesis. Archiv. Biochem. Biophys 1987; 258, 491-497

[19] Burton W.A., Scher M.G., Waechter C.J. Enzymatic phosphorylation of dolichol in central nervous tissues. J. Biol. Chem 1979; 254, 7129-7136 
[20] Szkopińska A., Nowak L, Swiezewska E., Palamarczyk G CTP-dependent lipid kinases of yeast. Archiv. Biochem. Biophys 1988; 266, 124-131

[21] Grabinska K, Palamarczyk G Dolichol biosynthesis in the yeast Saccharomyces cerevisiae: an insight into the regulatory role of farnesyl diphosphate synthase. FEMS Yeast Res 2002; 2:259-265.

[22] Hartley $M B$ and Imperiali B., At the membrane frontier: A prospectus on the remarkable evolutionary conservation of polyprenols and polyprenyl-phosphates. Archiv. Biochem. Biophys. 2012; 517, 83-97.).

[23] Jung, P. and Tanner, W Identification of the lipid intermediate in yeast mannan biosynthesis. Eur. J. Biochem 1973; 37, 16-21

[24] Szkopin.ska, A., Karst, F. and Palamarczyk G Products of S. cerevisiae cisprenyltransferase activity in vitro. Biochimie 1996; 78, 225-235

[25] Sagami, H., Igarashi, Y., Tateyama, S., Ogura, K., Roos, J. and Lennarz, W.J Enzymatic formation of dehydrodolichal and dolichal, new products related to yeast dolichol biosynthesis. J. Biol. Chem. 1996; 271, 9560-9566.

[26] Sagami, H.A., Kurisaki, A. and Ogura, K Formation of dolichol from dehydrodolichol is catalysed by NADPH-dependent reductase localised in microsomes of rat liver. J. Biol. Chem 1993; 268,10109-10113

[27] Warit S, Zhang N, Short A, Walmsley RM, Oliver SG, Stateva LI Glycosylation deficiency phenotypes resulting from depletion of GDP-mannose pyrophosphorylase in two yeast species. Mol Microbiol $2000 ; 36: 1156-66$.

[28] Zakrzewska A, Palamarczyk G, Krotkiewski H, Zdebska E, Saloheimo M, Penttilä M, Kruszewska JS Overexpression of the gene encoding GTP:mannose-1-phosphate guanyltransferase, mpg1, increases cellular GDP-mannose levels and protein mannosylation in Trichoderma reesei. Appl Environ Microbiol. 2003; 69:4383-4389.

[29] Fonzi,W.A, Irwin M.Y 1993; Isogenic strain construction and gene mapping in Candida albicans. Genetics. 134:717-28.

[30] Cantagrel V, Lefeber DJ, Ng BG, Guan Z, Silhavy JL, Bielas SL, Lehle L, Hombauer H, Adamowicz M, Swiezewska E, De Brouwer AP, Blümel P, Sykut-Cegielska J, Houliston S, Swistun D, Ali BR, Dobyns WB, Babovic-Vuksanovic D, van Bokhoven H, Wevers RA, Raetz CR, Freeze HH, Morava E, Al-Gazali L, Gleeson JG SRD5A3 is required for converting polyprenol to dolichol and is mutated in a congenital glycosylation disorder 2010; Cell. 142 (2):203-217.

[31] Mosch HU and Fink GR Dissection of filamentous growth by transposon mutagenesis in Saccharomyces cerevisiae 1997; Genetics 145(3):671-84

[32] Kruszewska, J., Butterweck, A., Kurzątkowski, W., Migdalski, A., Kubicek, C.P. and Palamarczyk, G. Overexpression of the Saccharomyces cerevisiae Mannosylphosphodolichol synthase -encoding gene in Trichoderma reesei results in the increased level of protein secretion and abnormal cell ultrastructure. Appl. Environ. Microbiol 1999; 65, 2382-2387.

[33] Perlińska-Lenart U, Kurzatkowski W, Janas P, Kopińska A, Palamarczyk G, Kruszewska JS.. Protein production and secretion in an Aspergillus nidulans mutant impaired in Glycosylation, Acta Biochim Pol. 2005; 52: 195-206. 
[34] Juchimiuk, M., Pasikowska, M., Zatorska, E., Laudy, A., Smoleńska-Sym, G., Palamarczyk, G. Defect in dolichol-dependent glycosylation increases sensitivity of Saccharomyces cerevisiae towards anti-fungal drugs. Yeast 2010; 27: 637-645.

[35] Orłowski, J., Machula, K., Janik, A., Zdebska, E., Palamarczyk,G Dissecting the role of dolichol in cell wall assembly in the yeast mutants impaired in early glycosylation reactions. Yeast 2007; 24: 239-252.

[36] Takeguchi, M., Kimala, Y., Kohno, K. Saccharomyces cerevisiae Rot1 is an essential molecular chaperone in the endoplasmic reticulum. Mol Biol Cell.; 2008; 19: 3514-3525.

[37] Machi K, Azuma M, Igarashi K, Matsumoto T, Fukuda H, Kondo A, Ooshima H Rot1p of Saccharomyces cerevisiae is a putative membrane protein required for normal levels of the cell wall 1,6-beta-glucan. Microbiology 2004; 150:3163-3173.

[38] Pasikowska M., Palamarczyk G, and Lehle L The essential endoplasmic reticulum chaperone Rot1 is required for protein $N$ - and O-glycosylation in yeast 2012; Glycobiology, accepted

[39] Girrbach V, Zeller T, Priesmeier M, Strahl-Bolsinger S Structure-function analysis of the dolichyl phosphate-mannose: protein O-mannosyltransferase ScPmt1p. 2000; J Biol Chem. 275:19288-19296.

[40] Ernst JF, Prill SK. O-glycosylation 2001; Med Mycol. 39 Suppl 1:67-74.

[41] Willger SD, Ernst JF, Alspaugh JA, Lengeler KB. Characterization of the PMT gene family in Cryptococcus neoformans. PLoS One. 2009; 4(7):e6321.

[42] Gentzsch M, Tanner W. Protein-O-glycosylation in yeast: protein-specific mannosyltransferases Glycobiology 1997; 7, 481-4866.

[43] Harty C, Strahl S, Römisch K O-mannosylation protects mutant alpha-factor precursor from endoplasmic reticulum-associated degradation. Mol Biol Cell 2001; 12:1093-10101.

[44] Prill SK, Klinkert B, Timpel C, Gale CA, Schröppel K, Ernst JF. PMT family of Candida albicans: five protein mannosyltransferase isoforms affect growth, morphogenesis and antifungal resistance. Mol Microbiol 2005; 55(2):546-560.

[45] Górka-Nieć W, Kania A, Perlińska-Lenart U, Smoleńska-Sym G, Palamarczyk G, Kruszewska JS Integration of additional copies of Trichoderma reesei gene encoding protein O-mannosyltransferase I results in a decrease of the enzyme activity and alteration of cell wall composition.. Fungal Biol. 2011; 115:124-32.

[46] Chandra J, Kuhn DM, Mukherjee PK, Hoyer LL, McCormick T, Ghannoum MA. Biofilm formation by the fungal pathogen Candida albicans: development, architecture, and drug resistance. J Bacteriol. 2001; 183(18):5385-94.

[47] Ramage G, Wickes BL, López-Ribot JL. Inhibition on Candida albicans biofilm formation using divalent cation chelators (EDTA). Mycopathologia. 2007;164(6):301-6. Epub 2007 Oct 2.

[48] Ramage G, López-Ribot JL. Techniques for antifungal susceptibility testing of Candida albicans biofilms Methods Mol Med. 2005; 118:71-79.

[49] Kojic EM, Darouiche RO. 2004. Candida infections of medical devices. Clin Microbiol Rev.;17(2):255-67.

[50] Uppuluri P, Pierce CG, López-Ribot JL.2009. Candida albicans biofilm formation and its clinical consequences. Future Microbiol. 2009; 4(10):1235-7 
[51] Silberstein S, Gilmore R. 1996 Biochemistry, molecular biology, and genetics of the oligosaccharyltransferase. FASEB J.;10(8):849-58.

[52] Dean, N. 1995. Yeast glycosylation mutants are sensitive to aminoglycosides. Proc Natl Acad Sci U S A 92:1287-1291.

[53] Zhou J, Zhang H, Liu X, Wang PG, Qi Q. 2007. Influence of N-glycosylation on Saccharomyces cerevisiae morphology: a golgi glycosylation mutant shows cell division defects. Curr Microbiol.;55(3):198-204.

[54] Bai C, Xu XL, Chan FY, Lee RT, Wang Y. 2006. MNN5 encodes an iron-regulated alpha1,2-mannosyltransferase important for protein glycosylation, cell wall integrity, morphogenesis, and virulence in Candida albicans.. Eukaryot Cell.;5 (2):238-247.

[55] Buurman ET, Westwater C., Hube B, Brown A.J., Odds FC, Gow N.A. 1998 Molecular analysis of CaMnt1p, a mannosyltransferase important for adhesion and virulence of Candida albicans. Proc. Natl. Acad. Sci. USA 95, 7670-7675.

[56] Bates S., MacCallum, D.M., Bertram, G., Munro, C.A., Hughes, H.B., BuurmanE.T., Brown A.J.P., Odds, F.C. and Gow, N.A.R. 2005 Candida albicans Pmr1p: a secretory pathway $\mathrm{Ca}^{2+} / \mathrm{Mn}^{2+}$ P-type ATPase is required for glycosylation and virulence. J. Biol. Chem. 2005 ;280(2):1051-60.

[57] Levin DE. 2005. Cell wall integrity signaling in Saccharomyces cerevisiae.. Microbiol Mol Biol Rev. 69(2):262-91.

[58] Hutzler F, Gerstl R, Lommel M, Strahl S. 2008 Protein N-glycosylation determines functionality of the Saccharomyces cerevisiae cell wall integrity sensor Mid2p. Mol Microbiol. 68(6):1438-49. Epub 2008 Apr 11. 